\title{
Variation in hydrogen cyanide production between different strains of Pseudomonas aeruginosa
}

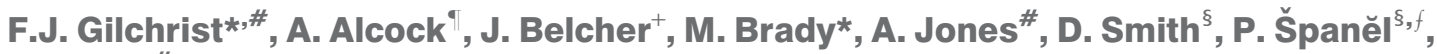 \\ K. Webb" and W. Lenney*,+
}

ABSTRACT: There is increasing interest in using the cyanogenic properties of Pseudomonas aeruginosa to develop a nonmicrobiological method for its detection. Prior to this, the variation in cyanide production between different $\boldsymbol{P}$. aeruginosa strains needs to be investigated.

Hydrogen cyanide (HCN) released into the gas phase by 96 genotyped $P$. aeruginosa samples was measured using selected ion flow tube-mass spectrometry after 24, 48, 72 and $96 \mathrm{~h}$ of incubation. The HCN produced by a range of non-P. aeruginosa cultures and incubated blank plates was also measured.

All $P$. aeruginosa strains produced more HCN than the control samples, which generated extremely low levels. Analysis across all time-points demonstrated that nonmucoid samples produced more HCN than the mucoid samples $(p=0.003)$, but this relationship varied according to strain. There were clear differences in the headspace HCN concentration for different strains. Multivariate analysis of headspace $\mathrm{HCN}$ for the commonest strains (Liverpool, Midlands_1 and Stoke-on-Trent, UK) revealed a significant effect of strain $(p<0.001)$ and a borderline interaction of strain and phenotype $(p=0.051)$.

This evidence confirms that all $\boldsymbol{P}$. aeruginosa strains produce HCN but to varying degrees and generates interest in the possible future clinical applications of the cyanogenic properties of P. aeruginosa.

\section{KEYWORDS: Microbiology, Pseudomonas aeruginosa}

$\mathbf{T}$ he opportunistic organism Pseudomonas aeruginosa is of particular significance in cystic fibrosis (CF) due to its association with increased morbidity and mortality [1]. Initial infection usually occurs with a nonmucoid strain that is sensitive to anti-pseudomonal antibiotics. Early, aggressive treatment can therefore result in eradication [2]. As the infection becomes chronic, the $P$. aeruginosa phenotype changes to mucoid, making eradication unlikely [3]. Although most patients are infected by their own individual $P$. aeruginosa strain, siblings often carry the same strain indicating infection from a common environmental strain or from crossinfection [4]. P. aeruginosa strains found in more than one patient (clonal strains), which are often multiple-antibiotic resistant and transmissible, have been identified in populations with $\mathrm{CF}$ in the UK and Australia [5-7]. This has had a major impact on the infection control precautions necessary for patients with $C F$ and further highlights the need for prompt, accurate $P$. aeruginosa diagnosis.
In children who are unable to expectorate sputum, current methods of $P$. aeruginosa diagnosis are unreliable (cough swab/cough plate) [8-10], unpleasant (induced sputum) [11] or invasive (bronchoscopy) [12]. There is an urgent need for a reliable, noninvasive method of diagnosing $P$. aeruginosa and there is significant interest in whether this could be based on the ability of $P$. aeruginosa to synthesise cyanide [13-15]. $P$. aeruginosa is one of a limited number of organisms to have this property and the only such organism frequently found in the respiratory tract of children with CF [16]. Using a cyanide ion-selective electrode to measure the $\mathrm{CN}^{-}$ions in aqueous solution, cyanide levels of $300-500 \mu \mathrm{M}$ have been found in $P$. aeruginosa cultures $[16,17]$ and of up to $130 \mu \mathrm{M}$ in the sputum of CF and non-CF bronchiectasis patients with $P$. aeruginosa infection [14, 15].

When hydrogen cyanide ( $\mathrm{HCN})$ is in aqueous solution at a neutral $\mathrm{pH}$ it is mainly undissociated, meaning it is readily released into the

\section{AFFILIATIONS}

*Academic Dept of Child Health,

"Dept of Microbiology, University Hospital of North Staffordshire,

${ }^{\S}$ Institute of Science and Technology in Medicine, Keele University, Guy Hilton Research Centre,

Stoke-on-Trent,

${ }^{*}$ Manchester Adult Cystic Fibrosis

Centre, Wythenshawe Hospital,

Manchester,

${ }^{+}$Institute for Primary Care and Health Sciences Research, Keele University, Keele, UK, and

${ }^{f}$ Dept of Chemical Physics,

J. Heyrovský Institute of Physical Chemistry, Prague, Czech Republic.

\section{CORRESPONDENCE}

F.J. Gilchrist

Academic Dept of Child Health

University Hospital of North

Staffordshire

Newcastle Road

Stoke-on-Trent ST4 6QG

UK

E-mail: fjgilchrist@hotmail.com

Received:

Oct 252010

Accepted after revision:

Jan 022011

First published online:

Jan 272011 
headspace. Selected ion flow tube-mass spectrometry (SIFTMS) has therefore been used to measure gas phase HCN. Using this methodology the HCN concentration has exceeded 17,000 parts-per-billion (ppb) by volume in the headspace (volume $200 \mathrm{~mL}$ ) over P. aeruginosa cultures [18] and $60 \mathrm{ppb}$ in the breath of CF patients infected with $P$. aeruginosa [13]. Conversely, cyanide is essentially absent from the sputum of CF and non-CF bronchiectasis patients without $P$. aeruginosa infection and from the sputum of healthy controls $[14,15]$. HCN is also very low or absent in the breath of healthy patients [13, 19]. One of the possible limiting factors in using the cyanogenic property of $P$. aeruginosa in the development of a diagnostic technology is that some cultures do not seem to produce significant levels of cyanide $[18,20]$. Similarly, a minority of breath and sputum samples from $P$. aeruginosa positive patients do not have detectable cyanide levels [13-15].

The ability of $P$. aeruginosa to employ quorum sensing and the subsequent relationship between cyanide concentration and bacterial load, supports the hypothesis that the observed variation in cyanide production is dependent on $P$. aeruginosa strain. Quorum sensing is the process by which organisms use extracellular molecules to regulate phenotypes in response to population density. Cyanide production by $P$. aeruginosa is regulated, in part, by quorum sensing, with high population densities inducing synthesis [21-23]. Despite this, cyanide levels have been shown to be independent of $P$. aeruginosa bacterial load when measured in the sputum of $\mathrm{CF}$ and non-CF bronchiectasis patients, suggesting cyanide production is dependent on the presence of specific $P$. aeruginosa strains [14]. This hypothesis is also supported by the finding that certain clonal strains of $P$. aeruginosa overproduce specific quorum-sensing regulated exoproducts [24]. This is the first reported study to assess the variation in cyanide production by different $P$. aeruginosa strains.

It is possible that the airway inflammation caused by $P$. aeruginosa infection results in cyanide production by leukocytes. This is supported in vitro by reports of leukocytes challenged with Staphylococcus epidermidis producing HCN $[25,26]$ and a recent study from the Australian Respiratory Early Surveillance Team for Cystic Fibrosis (AREST CF) team who found the neutrophil number in bronchoalveolar lavage (BAL) samples to be a predictor of cyanide concentration [27]. However, BAL neutrophil count is significantly higher in patients infected with $P$. aeruginosa compared with those with no infection or infection with other organisms [28] and previous studies have not identified cyanide in patients infected with organisms other than $P$. aeruginosa [13-15]. The high percentage of $P$. aeruginosa negative patients in whom cyanide was identified in the AREST CF study [27] is in contrast to previous papers and this is likely to be the reason why the authors were unable to use cyanide to differentiate between the patients who had $P$. aeruginosa infection and those they believed to be free from $P$. aeruginosa.

\section{METHODS}

The HCN concentration in the headspace over $P$. aeruginosa cultures was analysed using SIFT-MS. This technique combines fast-flow tube technology and quantitative mass spectrometry to allow accurate quantification of volatile compounds. Precursor ions, selected according to the compounds to be detected and quantified, are generated in a discharge ion source, mass selected by a quadrupole mass filter, and then injected as selected ionic species into fast-flowing helium carrier gas in a flow tube. The gas/headspace to be analysed is sampled directly into the carrier gas/precursor ion swarm via a calibrated capillary. The precursor ions react with the trace gases in the sample producing ions that are characteristic of the trace gas molecules present in the sample. These product ions, and the precursor ions and their hydrates can be detected by a downstream quadruple mass spectrometer. An on-line computer will immediately calculate the partial pressures of the trace gases in the air sample from the ion count rates [29, 30].

As part of a previous study looking at cross-infection between CF patients, all $P$. aeruginosa isolates obtained from CF patients between January and May 2007 had been genotyped [31]. These isolates were stored on Microbank ${ }_{\mathbb{}}$ cryogenic beads (Pro-lab Diagnostics Inc., Neston, UK) at $-70^{\circ} \mathrm{C}$ in our microbiology laboratory (Dept of Microbiology, University Hospital of North Staffordshire, Stoke-on-Trent, UK). For this study, eight new cultures were prepared from each original isolate. This was done by placing a bead from each isolate directly onto a blood agar plate (Oxoid Ltd, Basingstoke, UK) and plating it out. From this plate out, eight blood agar plate cultures were then prepared. Each of these eight plates were covered with a lid, sealed in plastic bags (total volume $\sim 200 \mathrm{~mL}$ ) and incubated at $37^{\circ} \mathrm{C}$. From each original isolate, the headspace over two new plates (referred to later as a paired P. aeruginosa cultures) were analysed at $24 \mathrm{~h}$, two at $48 \mathrm{~h}$, two at $72 \mathrm{~h}$ and two at $96 \mathrm{~h}$. During analysis the lid was removed from the plate whilst keeping the bag sealed. The plastic bag was pierced with a hypodermic needle attached directly to the input port of the SIFT-MS instrument. The accumulated HCN concentration was analysed for $100 \mathrm{~s}$ and the mean concentration over this time was recorded. $\mathrm{HCN}$ analysis was performed using the count rate of the product ion $\mathrm{H}_{2} \mathrm{CN}^{+}$generated using $\mathrm{H}_{3} \mathrm{O}^{+}$precursor ions as previously described [32]. After analysis the bacterial cultures were appropriately discarded.

Using the same methodology five control cultures of Streptococcus pneumoniae, Staphlococcus aureus, Moraxella catarrhalis, Haemophilus influenzae and Aspergillus fumigatus were each plated onto eight agar plates and analysed after the same periods of incubation. Chocolate agar plates (Oxoid Ltd) were used for the H. influenzae samples, Sabouraud agar (Oxoid Ltd) for the A. fumigatus samples and blood agar (Oxoid Ltd) for the others. 10 blank blood agar plates and five blank chocolate agar plates were also analysed after the same periods of incubation.

\section{Statistical methods}

In order to assess the reliability of the process the intraclass correlation (ICC) was calculated using a two way random effects model. As the raw data was heavily skewed and remained nonnormal even after a log transformation, the $95 \%$ confidence interval (CI) for the ICC was generated using 1,000 bootstrapped samples. The Shapiro-Wilks' test confirmed that the differences remained non-normal even after transformation of the original values. Consequently median differences between the paired samples at each of the four time-points were generated with corresponding $95 \%$ CI. In this way the magnitude of differences between the paired samples at each of the time-points could be assessed. 
The HCN concentration for the 96 pairs of $P$. aeruginosa cultures was then averaged at each time-point. A repeatedmeasures mulitvariate ANOVA (Wilks' lamda) was used to compare the overall distribution of $\mathrm{HCN}$ production between phenotypes (mucoid or nonmucoid). A two-way multivariate ANOVA was also adopted to assess the effect of the three commonest strains and phenotype across all time-points. In both cases a log transformation was used to ensure homogeneity of covariance matrices across the groups. Scheffé's post hoc test was then performed to identify differences between the mucoid and nonmucoid groups at each time-point and similarly between the three strains. These analyses were repeated on the ranked data as a kind of sensitivity analysis. Due to the non-normality and heterogeneity present, median summaries between the two groups at each time-point were generated.

\section{RESULTS}

\section{Background data}

Of the 98 genotyped $P$. aeruginosa samples that had been stored as part of the previous study, 96 were available for analysis. 48 samples were mucoid and 48 were nonmucoid. There were 26 different strains, 10 of which were clonal strains. Four of these had been previously described: Liverpool $(n=8)$, Midlands_1 $(n=19)$, Midlands_2 $(n=4)$ and Stoke-on-Trent $(n=15)$. The other six had not been previously descibed: clonal_1 $(n=4)$, clonal_2 $(n=5)$, clonal_3 $(n=2)$, clonal_4 $(n=3)$, clonal_5 $(n=3)$ and clonal_6 $(n=2)$. The remaining 16 genotypes were unique to individual patients; 10 were isolated on more than one occasion (sporadic_1: $\mathrm{n}=5$; sporadic_2: $\mathrm{n}=3$; sporadic_3: $\mathrm{n}=3$; sporadic_4: $\mathrm{n}=2$; sporadic_5: $\mathrm{n}=2$; sporadic_6: $\mathrm{n}=2$; sporadic_7: $\mathrm{n}=2$; sporadic_8: $n=2$; sporadic_9: $n=2$; and sporadic_10: $n=2$ ) and six on a single occasion (sporadic_11-16).

\section{Reliability of $\mathrm{HCN}$ concentration between paired $\mathrm{P}$. aeruginosa cultures}

The ICC $(95 \%$ CI) or the HCN measurement between the 96 pairs of $P$. aeruginosa cultures, was $0.97(0.96-0.98)$ at $24 \mathrm{~h}, 0.97$ $(0.96-0.98)$ at $48 \mathrm{~h}, 0.97(0.96-0.98)$ at $72 \mathrm{~h}$ and $0.96(0.95-0.98)$ at $96 \mathrm{~h}$. The median difference in the HCN concentration between the paired $P$. aeruginosa cultures at each of the four time-points was 3 ppb by volume (95\% CI -1-6), 3 ppb by volume (95\% CI 1-8), 9 ppb by volume (95\% CI -1-27) and $5.5 \mathrm{ppb}$ by volume (95\% CI -7-29).

\section{Headspace HCN concentrations for P. aeruginosa and controls cultures}

The incubated blank plates and the control cultures of S. pneumoniae, S. aureus, $M$. catarrhalis, $H$. influenzae and A. fumigatus produced extremely low levels of HCN at all time-points ( $<10 \mathrm{ppb}$ ). As expected the HCN was significantly higher in the headspace above the $P$. aeruginosa cultures (table 1).

\section{Headspace HCN concentration for mucoid and nonmucoid phenotypes}

The comparison of the headspace HCN concentrations between the mucoid and nonmucoid phenotypes suggested increased production by the nonmucoid samples (table 2). This difference was significant when analysed across all time-points (Wilks' lambda: $F 4,91=4.35, \mathrm{p}=0.003)$ and after $24 \mathrm{~h}$ only $(\mathrm{p}=0.008)$ when the time-points were analysed individually. The same conclusions were reached using the ranked data although the corresponding p-values were 0.018 and 0.007 , respectively. Interestingly when headspace $\mathrm{HCN}$ concentrations were split according to strain and phenotype (table 4), some strains had higher HCN for nonmucoid samples (Liverpool and Stoke-onTrent) and some for mucoid samples (Midlands_1).

\section{Headspace HCN concentration for different P. aeruginosa strains}

There are clear differences in the headspace HCN concentrations between different $P$. aeruginosa strains, although there is also variability within strains (table 3 ). Multivariate analysis of the effect of strain, phenotype and the interaction of both on the headspace $\mathrm{HCN}$ concentration above the three commonest strains (Liverpool, Midlands_2 and Stoke-on-Trent) was undertaken (table 4). This results showed a significant effect of strain (Wilks' lambda: $F 8,66=5.76, \mathrm{p}<0.001$ ), no effect from phenotype (Wilks' lambda: $F 4,33=0.78, \mathrm{p}=0.55$ ) and a borderline interaction of strain and phenotype (Wilks' lambda: $F 8,66=2.01$,

TABLE 1 Headspace hydrogen cyanide (HCN) concentrations for Pseudomonas aeruginosa (PA) cultures, control cultures and incubated blank plates

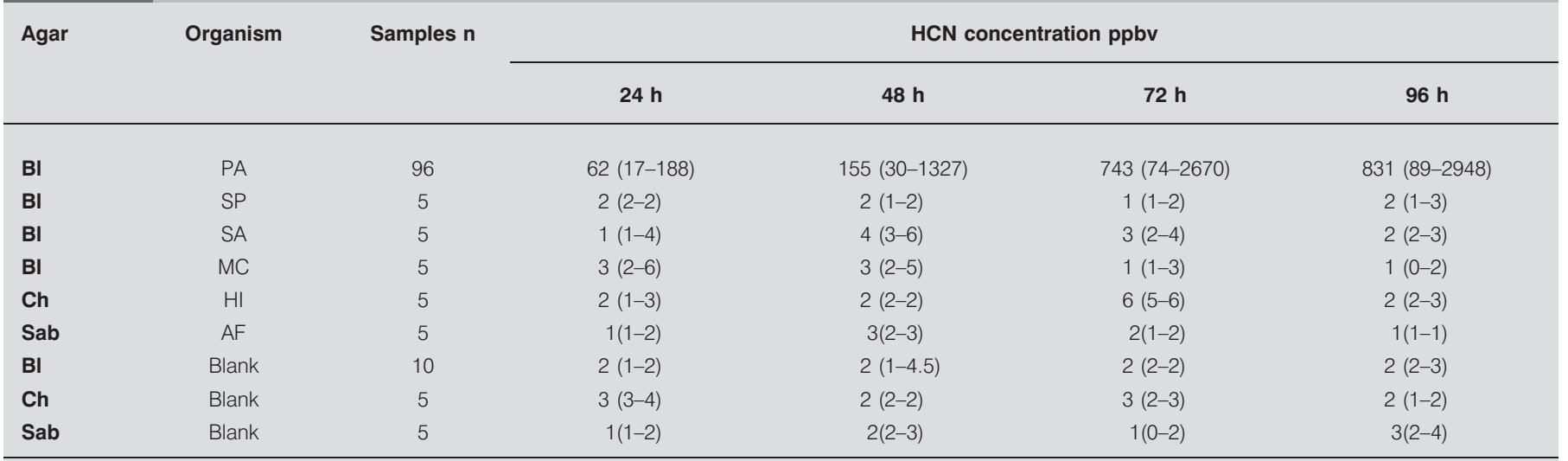

Data are presented as median (interquartile range), unless otherwise stated. ppbv: parts per billion by volume; BI: blood; Ch: chocolate; Sab: Sabouraud; SP Streptococcus pneumoniae; SA: Staphylococcus aureus; MC: Moraxella catarrhalis; HI: Haemophilus influenzae; AF: Aspergillus fumigatus. 
TABLE 2 Headspace hydrogen cyanide (HCN) concentrations for mucoid and nonmucoid Pseudomonas aeruginosa samples

\begin{tabular}{lcccrr} 
Phenotype & Samples n & \multicolumn{4}{c}{ HCN concentration ppbv } \\
\cline { 3 - 6 } & & $\mathbf{2 4} \mathbf{~ h}$ & $\mathbf{4 8 ~ h} \mathbf{~ h} \mathbf{~ h}$ & $\mathbf{9 6} \mathbf{~ h}$ \\
\hline Mucoid & 48 & $43(12-136)$ & $127(35-418)$ & $525(84-2234)$ & $747(91-3107)$ \\
Nonmucoid & 48 & $99(22-350)$ & $626(27-2279)$ & $2196(36-5528)$ & $1441(78-6429)$ \\
\hline
\end{tabular}

Data are presented as median (interquartile range), unless otherwise stated. ppbv: parts per billion by volume.

$\mathrm{p}=0.051)$. The lack of effect of phenotype is unsurprising as the Liverpool and Stoke-on-Trent strains had higher HCN concentrations above nonmucoid samples, whereas the Midlands_1 strain had higher concentrations above mucoid samples. Further analysis revealed the headspace HCN concentration above the Stoke-on-Trent strain to be significantly lower than the other two strains at all four time-points.

\section{DISCUSSION}

The present study is the first to investigate the variation in cyanide production between different strains of $P$. aeruginosa.
This is important as previous studies have been unable to detect cyanide in a proportion of $P$. aeruginosa samples, increasing the possibility that cyanogensis does vary according to $P$. aeruginosa strain $[18,20]$. We have identified HCN in the headspace of all the $P$. aeruginosa samples analysed, reflecting the sensitivity of the SIFT-MS instrument. Despite all the samples producing cyanide, there are clear differences in the quantity produced by different $P$. aeruginosa strains. The low levels of cyanide produced by the control cultures supports previous studies showing $P$. aeruginosa is one of a limited number of organisms to produce cyanide.

TABLE 3 Headspace hydrogen cyanide (HCN) concentrations for different Pseudomonas aeruginosa strains

Strain

Samples $n$
HCN concentration ppbv

\begin{tabular}{|c|c|c|c|c|c|}
\hline & & \\
\hline & & $24 \mathrm{~h}$ & $48 \mathrm{~h}$ & $72 \mathrm{~h}$ & $96 \mathrm{~h}$ \\
\hline Liverpool & 8 & $92(24-150)$ & $525(157-1397)$ & 2238 (483-3682) & $3093(2495-4501)$ \\
\hline Midlands_2 & 4 & $24(14-266)$ & $62(22-1261)$ & $199(71-1371)$ & $2186(222-4471)$ \\
\hline Stoke-on-Trent & 15 & $16(10-24)$ & $24(14-32)$ & $37(25-181)$ & $75(28-109)$ \\
\hline Clonal_1 & 4 & 499 (153-849) & 6570 (4281-8033) & 5852 (4858-7699) & 8727 (7505-8978) \\
\hline Clonal_4 & 3 & $169(145-174)$ & 776 (626-1029) & 7475 (7126-9128) & $7483(7382-8256)$ \\
\hline Clonal_5 & 3 & $13(12-15)$ & $22(21-29)$ & $39(26-44)$ & $89(61-97)$ \\
\hline Clonal_6 & 2 & $194(105-283)$ & $1084(564-1605)$ & $1979(1025-2933)$ & $3527(1826-5227)$ \\
\hline Sporadic_1 & 5 & $21(18-70)$ & 37 (35-124) & $46(34-1274)$ & $154(62-2301)$ \\
\hline Sporadic_2 & 3 & $16(12-35)$ & $27(15-27)$ & $167(87-252)$ & 588 (297-741) \\
\hline Sporadic_7 & 2 & 6406 (4231-8581) & 8299 (7460-9139) & 9174 (9012-9336) & 7453 (7124-7781) \\
\hline Sporadic_8 & 2 & $979(853-1105)$ & 5361 (3680-7052) & $6965(4928-9001)$ & $4432(2499-6364)$ \\
\hline Sporadic_9 & 2 & $45(34-56)$ & $117(90-143)$ & $424(255-593)$ & 789 (684-895) \\
\hline Sporadic_10 & 2 & 2716 (1389-4043) & $1037(830-1244)$ & 8342 (7819-8864) & $15714(14630-16799)$ \\
\hline Sporadic_11 ${ }^{\#}$ & 1 & 946 & 1285 & 3505 & 3585 \\
\hline Sporadic_12 ${ }^{\#}$ & 1 & 186 & 499 & 7559 & 12325 \\
\hline Sporadic_13" & 1 & 1002 & 5715 & 7621 & 5771 \\
\hline Sporadic_14 ${ }^{\#}$ & 1 & 113 & 1127 & 16612 & 11923 \\
\hline Sporadic_15 $5^{\#}$ & 1 & 738 & 2774 & 6861 & 12231 \\
\hline Sporadic_16 ${ }^{\#}$ & 1 & 7 & 31 & 75 & 34 \\
\hline
\end{tabular}

Data are presented as median (interquartile range), unless otherwise stated. ppbv: parts per billion by volume. ${ }^{\#}$ : as $\mathrm{n}=1$, absolute values are given. 


\begin{tabular}{|c|c|c|c|c|c|}
\hline TABLE 4 & $\begin{array}{l}\text { en cyanide } \\
\text { strains }\end{array}$ & oncentration & coid and non & d samples of th & pool, Midland_1 \\
\hline \multirow[t]{2}{*}{ Strain } & \multirow[t]{2}{*}{ Samples $\mathrm{n}$} & \multicolumn{4}{|c|}{ HCN concentration ppbv } \\
\hline & & $24 \mathrm{~h}$ & $48 \mathrm{~h}$ & $76 \mathrm{~h}$ & $92 \mathrm{~h}$ \\
\hline \multicolumn{6}{|l|}{ Liverpool } \\
\hline Nonmucoid & 5 & $123(62-216)$ & 949 (159-2740) & 3341 (3034-4505) & $4028(3104-5921)$ \\
\hline \multicolumn{6}{|l|}{ Midlands_1 } \\
\hline Mucoid & 14 & $127(71-184)$ & $182(138-368)$ & $1598(816-2753)$ & $804(725-2393)$ \\
\hline Nonmucoid & 5 & $58(17-85)$ & $87(16-1240)$ & $167(29-1079)$ & $544(68-2183)$ \\
\hline \multicolumn{6}{|l|}{ Stoke-on-Trent } \\
\hline Mucoid & 7 & $11(8-17)$ & $10(9-28)$ & $56(16-186)$ & 39 (28-97) \\
\hline
\end{tabular}

$P$. aeruginosa synthesises cyanide by the oxidative decarboxylation of glycine using a hydrogen cyanide synthase enzyme, encoded by the $h c n A B C$ gene locus. This process produces four electrons and four hydrogen atoms per glycine molecule. Temperatures of $34-37^{\circ} \mathrm{C}$ and microaerobic conditions $\left(\mathrm{O}_{2}\right.$ $<5 \%$ ) maximise $P$. aeruginosa cyanogenesis but it is inactivated by both atmospheric oxygen and strict anaerobic conditions $[16,17,33]$. P. aeruginosa avoids the toxic effects of cyanide on aerobic respiration by active detoxification mechanisms and synthesis of a respiratory chain terminated by a terminal oxidase insensitive to cyanide [34-37]. P. aeruginosa may produce cyanide to exclude other pathogens, thereby allowing it to become the dominant organism. This hypothesis is supported by a study showing cyanide to be the mediating factor in the paralytic killing model of Caenorhabditis elegans by $P$. aeruginosa [38]. The high concentrations of cyanide identified in the sputum and breath of CF patients [13-15] may also affect lung cellular function contributing to the lung damage caused by $P$. aeruginosa infection.

Traditionally, the organisms other than P. aeruginosa known to be cyanogenic are: Pseudomonas fluorescens, Pseudomonas aureofaciens, Pseudomonas chlororaphis, Chromobacterium violaceum and Rhizobium leguminosarum [16, 39]. These organisms are not usually isolated from patients with CF. More recently Burkholderia cepacia complex have also been shown to produce cyanide under biofilm and colonial growth conditions [40]. These are pathogens in the lungs of patients with CF but as they are usually acquired late in the course of the disease they are uncommon in children. Previously, the proposed cut-off for the HCN detected in the headspace over a culture to confirm $P$. aeruginosa was 100 ppb (sensitivity 68\% and specificity 100\%) [18]. These data suggest that for the cultures included in this study, using the highest HCN concentration at any of the time-point, the cut-off could be reduced to $10 \mathrm{ppb}$ (sensitivity $100 \%$ and specificity $100 \%$ ).

The excellent correlation between the HCN produced by the pairs of $P$. aeruginosa cultures confirms the reproducibility of cyanide production by $P$. aeruginosa isolates and of the SIFT-MS real-time analyses. Although there is variation in the $\mathrm{HCN}$ production by samples of the same strain, overall there are clear differences between $P$. aeruginosa strains. This is the first study to show this difference. We have also shown that all the $P$. aeruginosa samples produced detectable levels of $\mathrm{HCN}$, which reflects the high sensitivity and reliability of the SIFT-MS analyses. It is unclear how in vitro cyanide production by $P$. aeruginosa cultures correlates with in vivo cyanide detection in patient's breath or sputum. Specifically, as in vivo cyanide levels tend to be lower [13-15], it is not known if current methods are sensitive enough to detect cyanide in sputum or exhaled breath produced by a $P$. aeruginosa strain with lower cyanide production. It is also possible that the same strain of $P$. aeruginosa could produce different amounts of $\mathrm{HCN}$ depending on its position in the respiratory tract and the specific environmental conditions present.

Regarding phenotype, previous studies have reported higher cyanide production by mucoid $P$. aeruginosa cultures [20, 27]. Our data suggest the opposite, with nonmucoid cultures having higher $\mathrm{HCN}$ concentrations. This may be related to the timing of analysis, as the trend for $\mathrm{HCN}$ concentration was still increasing at $96 \mathrm{~h}$ for the mucoid samples, whereas the nonmucoid samples peaked at $72 \mathrm{~h}$ and started to fall at $96 \mathrm{~h}$. We may therefore have observed a different effect of phenotype if HCN analysis was undertaken after a longer period of incubation. Interestingly, when the $\mathrm{HCN}$ concentrations were analysed for strain and phenotype (table 4), the effect of phenotype seemed to differ between strains. The observed difference in the effect of phenotype on cyanogenesis between this and other studies may therefore be explained by which $P$. aeruginosa strain was included in the various studies.

In conclusion, the present study has demonstrated that all of our $P$. aeruginosa samples produced cyanide, but that the relative levels varied according to strain. This will create interest in the possible future clinical applications of the cyanogenic property of $P$. aeruginosa.

\section{STATEMENT OF INTEREST}

None declared. 


\section{REFERENCES}

1 Emerson J, Rosenfeld M, McNamara S, et al. Pseudomonas aeruginosa and other predictors of mortality and morbidity in young children with cystic fibrosis. Pediatr Pulmonol 2002; 34: 91-100.

2 Valerius NH, Koch C, Høiby N. Prevention of chronic Pseudomonas aeruginosa colonisation in cystic fibrosis by early treatment. Lancet 1991; 338: 725-726.

3 Govan JR, Deretic V. Microbial pathogenesis in cystic fibrosis: mucoid Pseudomonas aeruginosa and Burkholderia cepacia. Microbiol Rev 1996; 60: 539-574.

4 Renders NH, Sijmons MA, van Belkum A, et al. Exchange of Pseudomonas aeruginosa strains among cystic fibrosis siblings. Res Microbiol 1997; 148: 447-454.

5 Cheng K, Smyth RL, Govan JR, et al. Spread of beta-lactamresistant Pseudomonas aeruginosa in a cystic fibrosis clinic. Lancet 1996; 348: 639-642.

6 Jones AM, Govan JR, Doherty CJ, et al. Spread of a multiresistant strain of Pseudomonas aeruginosa in an adult cystic fibrosis clinic. Lancet 2001; 358: 557-558.

7 Armstrong DS, Nixon GM, Carzino R, et al. Detection of a widespread clone of Pseudomonas aeruginosa in a pediatric cystic fibrosis clinic. Am J Respir Crit Care Med 2002; 166: 983-987.

8 Rosenfeld M, Emerson J, Accurso F, et al. Diagnostic accuracy of oropharyngeal cultures in infants and young children with cystic fibrosis. Pediatr Pulmonol 1999; 28: 321-328.

9 Maiya S, Desai M, Baruah A, et al. Cough plate versus cough swab in patients with cystic fibrosis; a pilot study. Arch Dis Child 2004; 89: $577-579$.

10 Chavasse RJ, Cordle R, Petkar H. Cough plates for microbiological surveillance in cystic fibrosis. Arch Dis Child 2007; 92: 279.

11 De Boeck K, Alifier M, Vandeputte S. Sputum induction in young cystic fibrosis patients. Eur Respir J 2000; 16: 91-94.

12 Brennan S, Gangell C, Wainwright C, et al. Disease surveillance using bronchoalveolar lavage. Paediatr Respir Rev 2008; 9: 151-159.

13 Enderby B, Smith D, Carroll W, et al. Hydrogen cyanide as a biomarker for Pseudomonas aeruginosa in the breath of children with cystic fibrosis. Pediatr Pulmonol 2009; 44: 142-147.

14 Ryall B, Davies JC, Wilson R, et al. Pseudomonas aeruginosa, cyanide accumulation and lung function in CF and non-CF bronchiectasis patients. Eur Respir J 2008; 32: 740-747.

15 Sanderson K, Wescombe L, Kirov SM, et al. Bacterial cyanogenesis occurs in the cystic fibrosis lung. Eur Respir J 2008; 32: 329-333.

16 Blumer C, Haas D. Mechanism, regulation, and ecological role of bacterial cyanide biosynthesis. Arch Microbiol 2000; 173: 170-177.

17 Zlosnik JEA, Williams HD. Methods for assaying cyanide in bacterial culture supernatant. Lett Appl Microbiol 2004; 38: 360-365.

18 Carroll W, Lenney W, Wang T, et al. Detection of volatile compounds emitted by Pseudomonas aeruginosa using selected ion flow tube mass spectrometry. Pediatr Pulmonol 2005; 39: 452-456.

19 Enderby B, Lenney W, Brady M, et al. Concentrations of some metabolites in the breath of healthy children aged 7-18 years measured using selected ion flow tube mass spectrometry (SIFTMS). J Breath Res 2009; 3: 036001.

20 Carterson AJ, Morici LA, Jackson DW, et al. The transcriptional regulator AlgR controls cyanide production in Pseudomonas aeruginosa. J Bacteriol 2004; 186: 6837-6844.

21 Williams $\mathrm{P}$, Winzer $\mathrm{K}$, Chan WC, et al. Look who's talking: communication and quorum sensing in the bacterial world. Philos Trans R Soc Lond B Biol Sci 2007; 362: 1119-1134.
22 Girard G, Bloemberg GV. Central role of quorum sensing in regulating the production of pathogenicity factors in Pseudomonas aeruginosa. Future Microbiol 2008; 3: 97-106.

23 Bjarnsholt $T$, Givskov $M$. The role of quorum sensing in the pathogenicity of the cunning aggressor Pseudomonas aeruginosa. Anal Bioanal Chem 2007; 387: 409-414.

24 Salunkhe P, Smart CHM, Morgan JAW, et al. A cystic fibrosis epidemic strain of Pseudomonas aeruginosa displays enhanced virulence and antimicrobial resistance. J Bacteriol 2005; 187: 4908-4920.

25 Stelmaszyńska T. Formation of $\mathrm{HCN}$ and its chlorination to $\mathrm{ClCN}$ by stimulated human neutrophils -2 . Oxidation of thiocyanate as a source of HCN. Int J Biochem 1986; 18: 1107-1114.

26 Stelmaszyńska T. Formation of HCN by human phagocytosing neutrophils - 1. Chlorination of Staphylococcus epidermidis as a source of HCN. Int J Biochem 1985; 17: 373-379.

27 Stutz MD, Gangell CL, Berry LJ, et al. Cyanide in bronchoalveolar lavage is not diagnostic for Pseudomonas aeruginosa in children with cystic fibrosis. Eur Respir J 2011; 37: 553-558.

28 Douglas TA, Brennan S, Gard S, et al. Acquisition and eradication of $P$. aeruginosa in young children with cystic fibrosis. Eur Respir J 2009; 33: 305-311.

29 Amann A, Smith D, eds. Breath Analysis for Clinical Diagnosis and Therapeutic Monitoring. World Scientific Publishing Company, 2005. http:/ / eproceedings.worldscinet.com/9789812701954/ toc.shtml. Date last accessed: May 31, 2011.

30 Smith D, Spanel P. Selected ion flow tube mass spectrometry (SIFT-MS) for on-line trace gas analysis. Mass Spectrom Rev 2005; 24: 661-700.

31 Gilchrist FJ, Enderby B, Panagea S, et al. Transmission of Pseudomonas aeruginosa between patients with cystic fibrosis despite clinic separation. Midlands Med 2009; 25: 222-225.

32 Spanĕl P, Wang T, Smith D. Quantification of hydrogen cyanide in humid air by selected ion flow tube mass spectrometry. Rapid Commun Mass Spectrom 2004; 18: 1869-1873.

33 Worlitzsch D, Tarran R, Ulrich M, et al. Effects of reduced mucus oxygen concentration in airway Pseudomonas infections of cystic fibrosis patients. J Clin Invest 2002; 109: 317-325.

34 Zlosnik JEA, Tavankar GR, Bundy JG, et al. Investigation of the physiological relationship between the cyanide-insensitive oxidase and cyanide production in Pseudomonas aeruginosa. Microbiology 2006; 152: 1407-1415.

35 Cipollone R, Ascenzi P, Frangipani E, et al. Cyanide detoxification by recombinant bacterial rhodanese. Chemosphere 2006; 63: 942 949.

36 Cunningham L, Pitt M, Williams HD. The cioAB genes from Pseudomonas aeruginosa code for a novel cyanide-insensitive terminal oxidase related to the cytochrome bd quinol oxidases. Mol Microbiol 1997; 24: 579-591.

37 Cunningham L, Williams HD. Isolation and characterization of mutants defective in the cyanide-insensitive respiratory pathway of Pseudomonas aeruginosa. J Bacteriol 1995; 177: 432-438.

38 Gallagher LA, Manoil C. Pseudomonas aeruginosa PAO1 kills Caenorhabditis elegans by cyanide poisoning. J Bacteriol 2001; 183: 6207-6214.

39 Williams HD, Zlosnik JEA, Ryall B. Oxygen, cyanide and energy generation in the cystic fibrosis pathogen Pseudomonas aeruginosa. Adv Microb Physiol 2007; 52: 1-71.

40 Ryall B, Lee X, Zlosnik JEA, et al. Bacteria of the Burkholderia cepacia complex are cyanogenic under biofilm and colonial growth conditions. BMC Microbiol 2008; 8: 108. 